\title{
Efficiency in Electrical Heating Systems: an MAS Real World Application
}

\author{
José R. Villar, Roberto Pérez, Enrique de la Cal and Javier Sedano \\ Departamento Informática y Automática \\ Universidad de Oviedo \\ Campus de Viesques s/n 33204 Gijón, Spain \\ villarjose@uniovi.es; UO24411@uniovi.es; delacal@uniovi.es; jsedano@ubu.es
}

\begin{abstract}
In electrical heating systems, the electrical power consumption should be lower than the Contracted Power Limit. Energy distribution devices are used to solve this problem, but they are only concerned with the electrical energy. We claim that this energy distribution must also consider the comfort level in the building. In this work, an electrical energy distribution MAS for coordinating the electrical heaters is proposed. The MAS is responsible for both objectives: the electrical power must be lower than the Contracted Power Limit and the comfort level in the building must be maintained if sufficient power is available. The MAS is now being implemented in a real world application of an electrical heating system marketed by a local company.
\end{abstract}

Keywords: MAS applications, Software Agents in Industry, Energy distribution, Electrical heating systems.

\section{Introduction}

In Spain, a local company marketed a new catalogue of dry electrical heaters in the middle of the year 2008. The total power installed in a domestic electrical heating system in Spain easily surpasses $7 \mathrm{~kW}$. When electrical heaters are used, the electrical energy consumption could be higher than the common contracted power limit (for short, CPL). Typically, an energy distribution device shares the electrical energy between the electrical heaters, avoiding surpassing the CPL. The main drawback is that it does not consider the comfort level in the house.

To improve the efficiency of the electrical heating system, the local company desires to design a Central Control Unit (CCU) in order to save energy and distribute it among the heaters in a building while maintaining the comfort level in the house. In previous works, the development of such a device has been analyzed, and a multi agent hybrid fuzzy system has been proposed [15, 14, 16, 13]. The solution integrates the electric heaters and the CCU. The CCU is responsible for distributing the available electric energy to the heaters based on the energy balance concept and with the objective of keeping the user defined comfort level in the house. Although the proposal has proved successful in the suitable distribution of the available electric energy, it suffers from some deficiencies. Specifically, some bias was found in the steady state. Also, there was a lack of stability in the electric energy output due to fluctuations that must be faced. 
In this work, a solution to the electrical energy distribution for the electrical heating system is described. An MAS architecture has been adopted, with the $\mathrm{CCU}$ and the heaters collaborating to reach both objectives: to keep the power consumption lower than the CPL and to maintain the comfort level in the building. The energy distribution algorithm, which makes use of a Fuzzy Rule Base System (FRBS), and the MAS design are detailed.

This work is organized as follows. The next section deals with the Spanish regulations and the problem definition. Section 3 details the MAS proposed in this work. Implementation aspects and experimentation results are given in Section 4. Finally, conclusions and future work are presented.

\section{Preliminaries}

In the first quarter of 2007, the Spanish parliament approved a new building regulation [5]. As a result, building methods have been updated [6]. This new regulation had many consequences, as it determined how new buildings must be accomplished [4]: materials, isolation, energy efficiency, ventilation rates, etc. In Spain, the LIDER software [7] has been developed and should be used to calculate the heating installation in a building: the number of heaters and their nominal power are fixed.

The RITE establishes 5 climate winter zones, named with a letter from A to E, where E represents the maximum in weather severity. A peculiar fact [5] is that in Spain only 3 of the 5 winter zones defined in the RITE are considered. Moreover, a number between 1 and 5, related with the summer weather severity, is also given. The combination of winter and summer severities determines the climate zone for each location in Spain.

Furthermore, the constructors build many different kinds of buildings: condominiums -each apartment includes 2, 3 or 4 bedrooms-, detached and terraced houses, etc. All of them can have an electrical heating system installed, so the design of an energy distribution device must consider all the possible cases. The term building topologies refers to all of the building parameters that influence the heating system. Such parameters include the type of house, the geometrical aspects, the inner partition, the materials, etc. For example, the building envelope could help in reducing the heating losses. The building topologies have been extracted from the analysis of the building market, and have been reported in [13, 16]. The proposed building topologies should be used to validate any proposal of an electrical energy distribution for electrical heating systems.

\subsection{The Problem Description}

The main goal is the design of a heating system to distribute the available power without exceeding the CPL. The predefined comfort level in the house must also be reached. The heating system comprises the electrical heaters installed in all of the rooms of a house and the CCU. The range of the nominal powers for the 
heaters are 500,1000, 1200 and 1500 Watts. The houses must comply with the Spanish regulations.

The inhabitants establish the comfort level in the house or in a room by setting the predefined suitable environmental variables. In the case of this project, only the temperature in each room is considered due to economic reasons. Future works should consider other measurements, such as the humidity percentage. The different room temperatures are to be measured using the temperature sensors included in each heater. Although the temperatures measured in the heaters are noisy, a pre-process module is carried out in order to improve the measurement quality; the heaters designers have introduced this pre-process module.

Even though there are devices for energy distribution -specifically, energy rationalizers-, these devices distribute the energy consumption but do not consider the comfort level in the house. Consequently, it is necessary to introduce a new device, called CCU, which will be responsible for the saving and distribution of energy to the installed heaters; all of the devices will collaborate to achieve the goal. A measure of the current power consumption is needed to maintain the electrical power consumption below the CPL.

Some hints about costs are to be considered when deciding the system architecture. Firstly, the communications between devices should be wireless in order to reduce the cost of the installation, specifically, the communications must be managed by a Zigbee wireless network [10]. Secondly, the cost of configuring the system should also be low: the installer must configure the whole system in a limited period of time. The configuration must be as simple as possible, with a reduced set of parameters.

\section{The MAS approach for electrical energy distribution}

An MAS architecture based on a Zigbee network [10] has been proposed as a solution for the electrical energy distribution. In this approach, both the heaters and the CCU act as agents, collaborating to reach the goals. This MAS solution is based on several reasons. Firstly, the use of a wireless network such as Zigbee reduces the installation cost. Moreover, the robustness of the solution is improved as the electrical heaters agents can behave in a stand-alone mode when the distributed system collapses. Nevertheless, the CCU is responsible for the electrical energy distribution when the distributed system is up and the heaters collaborate with the CCU carrying out several necessary tasks. Finally, the system must be implemented in language $\mathrm{C}$ for microcontrollers, so the modules should not be of high computational complexity.

In order to describe the whole solution this section is organized as follows. First, the MAS system is outlined, and then a short description of the energy distribution algorithm (EDA) is presented, concluding with the MAS architecture and the agents' descriptions. 


\subsection{The electrical energy distribution system}

The concept of energy balance is used in the multi-agent system outlined in Figure 1. In short, the heaters send the CCU the temperature of the room and the heating energy error. The heating energy error is calculated as the difference between the estimated required energy to reach the comfort level in the room and the energy that has been spent in heating the room (heating energy). The CCU measures the instantaneous consumed current in the house and estimates the power that can be spent on heating the house (available power). The CCU also stores the set point temperature profiles for all the rooms in the house, and the association between rooms and heaters. Finally, the CCU carries out the Energy Distribution Algorithm (EDA) to distribute the instantaneous power (heating power) for each heater. The heating power is the fraction of the nominal power that a heater is allowed to spend; the heating energy is the heating power by time unit.

The EDA makes use of an FRBS (a fuzzy controller-FRBS-1-) to calculate the percentage of nominal power for a heater given the room temperature error and the energy error. The temperature error is calculated as the difference between the temperature set point and the room temperature. Fuzzy logic is chosen to manage the wide variety of topologies that the system must work with: the same algorithm must ensure the power distribution and the energy saving for many different cases. An FRBS is a Fuzzy inference system that allows to model with human interpretability [2]. An FRBS includes a Knowledge Base with two main parts: the

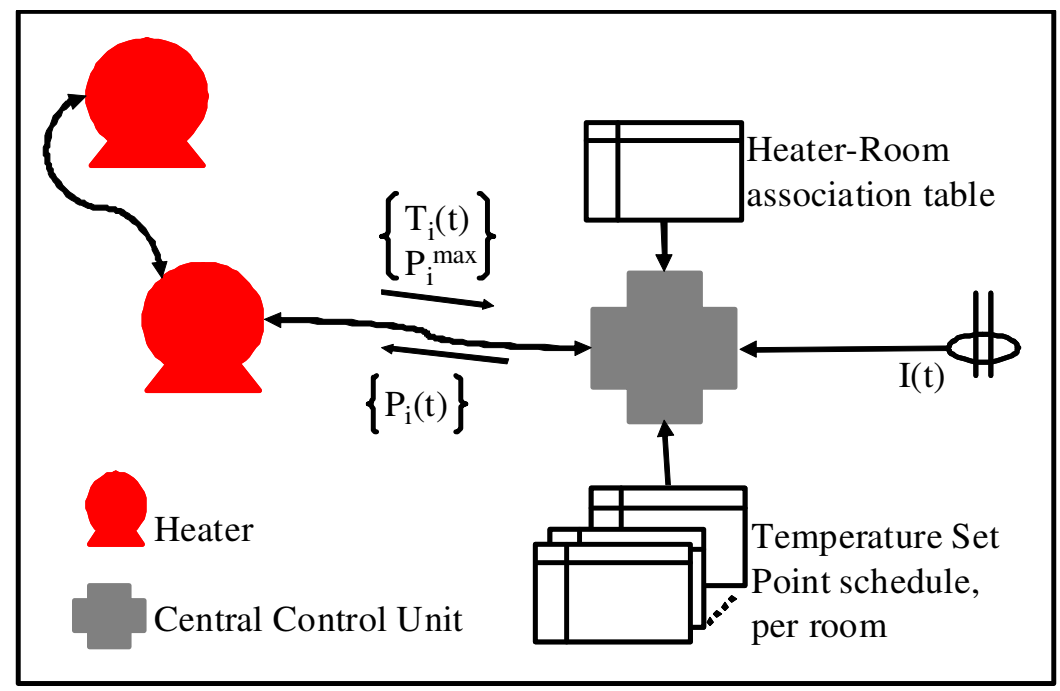

Fig. 1 The multi-agent system: the involved agents.

fuzzy rule base and the database. The former includes the rules and the relationships between the fuzzy partitions of each variable. The latter comprises the description of each membership function for each fuzzy partition. 
A block diagram of the whole process is shown in Figure 2. There are two stages in the solution: the Design Stage and the Run Stage. In the Design Stage, an FRBS-1 is generated for each pair of climate zone and building topology (configuration). To generate the learning datasets for the FRBS-1 the simulation software tool HTB2 [11] has been used.

In the Run Stage the heating power for each heater is calculated using the concept of the balance of energy: the heating energy must equal the required energy in order to reach the comfort in the house. The available power is distributed between the heaters according to their percentages of nominal power. The heater agents calculate the energy error and the temperature in the room and send them to the CCU. The CCU uses that information, the power consumption in the house and the temperature set point profiles to distribute the available power. Also, the CCU uses an FRBS-1 for each room: according to the configuration, an initial FRBS-1 is chosen. The CCU can modify an FRBS-1 in order to improve the energy distribution.

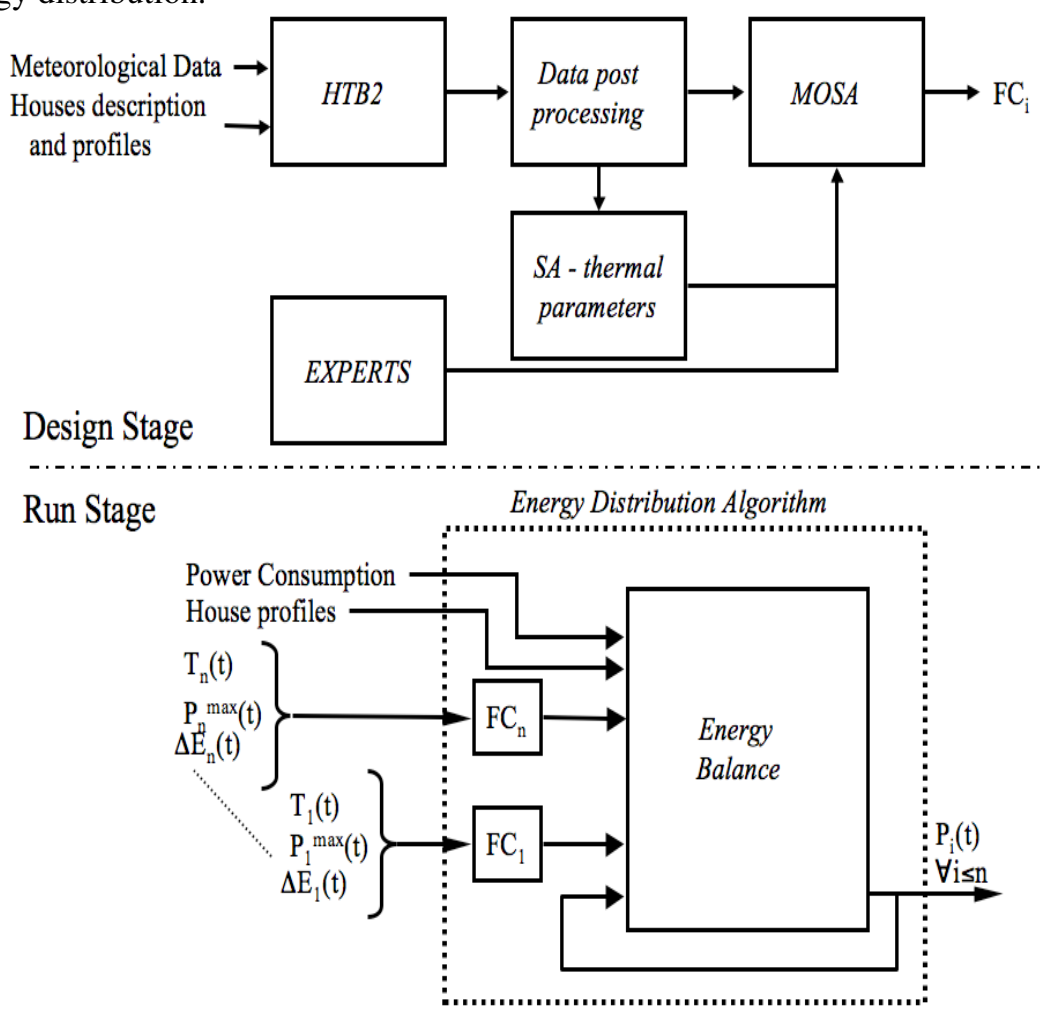

Fig. 2 The two steps procedure for distributing the power between the heaters

In the Design Stage two learning algorithms are used. A simulated annealing algorithm is used to learn the thermal dynamics of each room in order to train the FRBS-1. A multi-objective simulated annealing (MOSA) is used to train the FRBS-1 attending to the main objectives: to avoid surpassing the CPL in the 
house and to maintain the comfort level in the house if there is enough available power. A Michigan approach is used [8], and the initial individual is given by the company experts. Future work must include testing different FRBS learning approaches in the literature [1].

\subsection{The EDA description}

The power distribution among the heaters is carried out using the concept of the balance of energy. This means that the energy spent on heating the house must equal the energy estimated as required. Each time the energy distribution is needed the algorithm in Figure 3 is run. The required energy is estimated locally by the heaters, which send it to the CCU. Also, the CCU receives from each heater the temperature in the room. The CCU then aggregates both measures for each room in the building according to the number of heaters installed in it.

The CCU calculates the available power $(\mathrm{Pa})$ as the difference between the CPL and the current consumption due to the small power devices and lighting. The FRBS-1 from room $\mathrm{i}$ is then used to propose the heaters heating power (Phi). Finally, the $\mathrm{Pa}$ is distributed between the heaters according to their Phi, which is updated. This Phi is the heating power that the heater $i$ is allowed to spend on the next duty cycle. The last step in this algorithm is to join the switch ON time slots assigned to each heater to avoid fluctuation in the power output.

\subsection{The MAS architecture}

The MAS architecture is based on two kinds of agents: the CCU agent -which acts as a coordinator- and the heater agent -which acts as data provider and actuation unit-. Both types of agents collaborate to distribute the power without surpassing the CPL while maintaining the comfort level in the house if there is sufficient available power.

An MAS paradigm is considered to provide the heating system with a robust behavior, as proposed in [3]. The MAS methodology used is that resembled in [9] [12]. The MAS runs over a Zigbee wireless network. The EDA -distributed between the CCU and the heaters- directs the power output of all the heaters when the network is up. When the network is down, or when the CCU is out of service, all the heaters must act as normal stand-alone heaters until the system recovers. Figure 4 shows the schema of the MAS, where the data flows between heaters and $\mathrm{CCU}$ and some of the agent behaviors are presented.

\section{The CCU agent}

The $\mathrm{CCU}$ agent is responsible for managing the building information: the building topology, the climate zone, the association table between heaters and rooms and the temperature set point profiles for each room. Also, the CCU measures the power consumption in the building in order to calculate the available power.

In the Run Stage, the CCU runs the EDA, distributing the power and synchronizing all the heaters in the building. 


\section{The Electrical Heater agent}

The electrical heater agent is responsible for heating the room where its respective heater is installed. This agent has the ability to detect the collapse of the distributed system (the CCU breaks down, the wireless network is OFF, etc.). Also, it will automatically change to the collaborative work if the system recovers.

This agent is responsible for some important tasks, such as calculating the temperature in each room or estimating the required power. Also, some behaviors like open window detection or the initiation of the association to a CCU correspond to this agent.

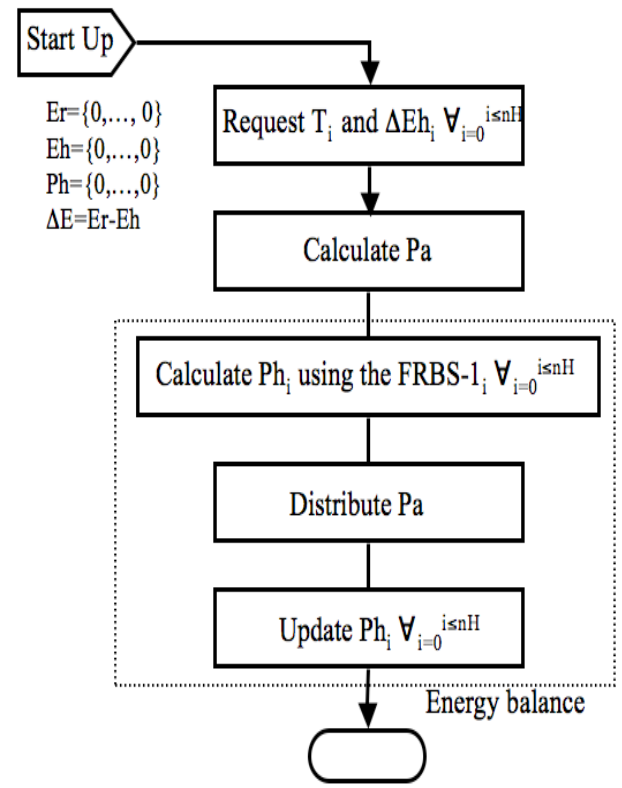

Fig. 3 The EDA iteration flowchart.

\section{The implementation of the MAS approach}

The MAS described in the previous section is now being implemented in micro-controller devices. The CCU includes the FRBS-1, the EDA, the rest of the pre-defined behaviours and a human-machine interface (HMI) to set up the system. This HMI is text based to simplify the code needed and to reduce the cost of the CCU unit in the market. 


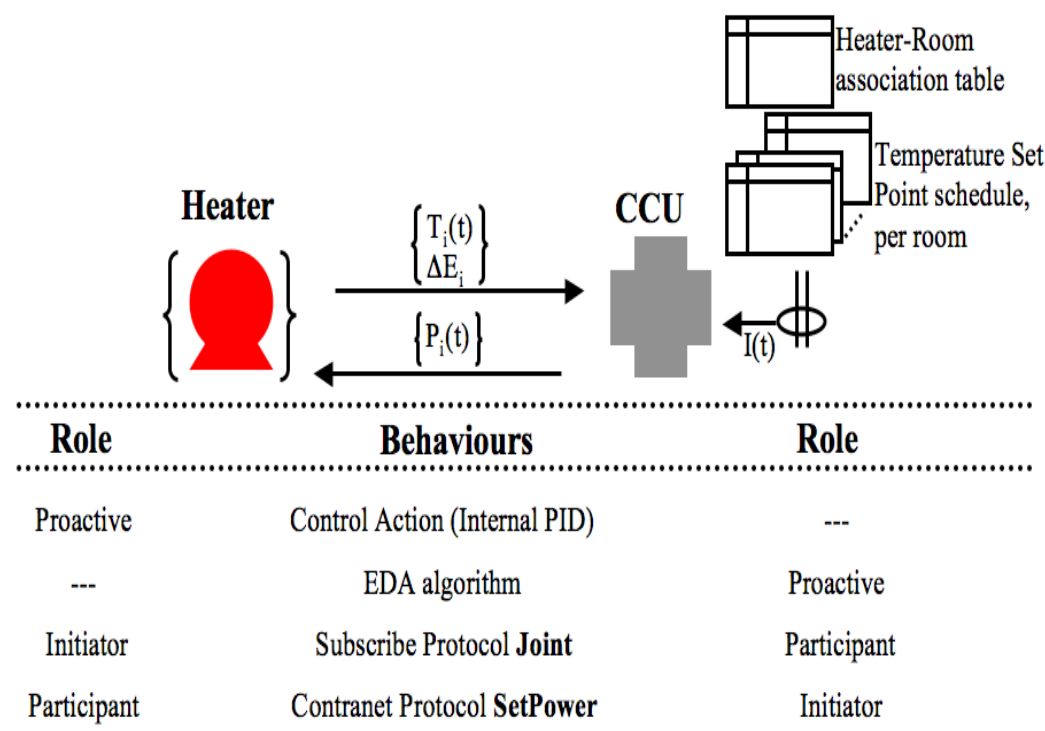

Fig. 4 The MAS architecture and some of the behaviours: the role of each type of agents is included.

Also, the heater agents have an HMI, which is also text based. This HMI allows users to configure the heater in the stand-alone mode and to set the network address in case of being integrated within the energy distribution system.

The CCU needs a small set of parameters to be configured: the climate zone and the building topology. Also, the temperature set up profile for each room and for the building must be given, but this is common to all heating system controllers. Finally, a procedure of discovering the heaters from the CCU and associating them to a room of the building must be executed.

In Figure 5 the photo of the prototype being developed is included. The CCU is the micro-controller device on the left; the remaining devices are the heaters. The centred heater includes the HMI. The Atmel ATAVRRZ200 Zigbee demonstration kit is being used to develop the prototype.

\section{Conclusions and future works}

A valid approach to distribute the available electrical power between the heaters in an electrical heating system has been developed. An MAS approach has proven as valid for these purposes, providing robustness and simplicity to the distributed heating system. The HAIS proposed for the design stage is able to extract the information and to successfully train the FRBS-1.

Future work includes validating the prototype in real situations previous to manufacturing a marketable product. Also, the HAIS presented in this work must be tested against different FRBS learning approaches available in the literature in order to obtain better fuzzy controllers. 


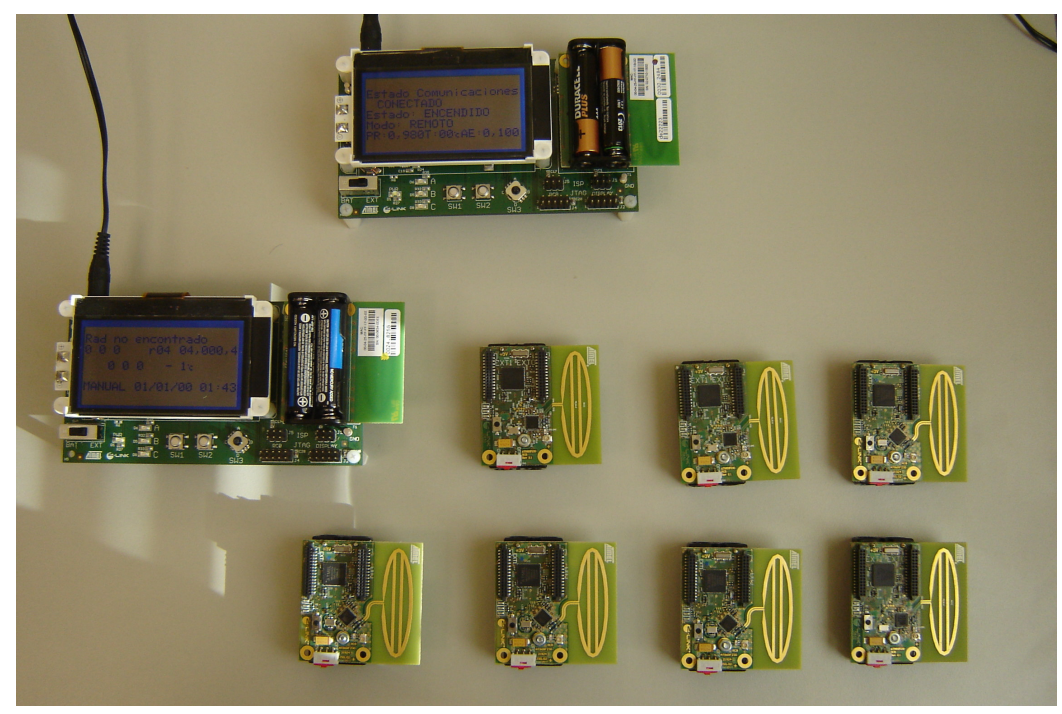

Fig. 5 The MAS prototype: the left-most micro-device is the CCU, while the remaining devices are heaters.

Acknowledgements. This research work has been funded by Gonzalez Soriano, S.A. -by means of the CN-08-028-IE07-60 FICYT research project- and by the Spanish Min. of Education, under the grant TIN2005-08386-C05-05.

\section{References}

[1] J. Alcalá-Fdez, L. Sánchez, S. García, M.J. del Jesus, S. Ventura, J.M. Garrell, J. Otero, C. Romero, J. Bacardit, V.M. Rivas, J.C. Fernández, and F. Herrera. Keel: A software tool to assess evolutionary algorithms to data mining problems. Soft Computing, 2007. In press, doi: 10.1007/s00500-008-0323-y.

[2] J. Casillas, O. Cordón, F. Herrera, and P. Villar. A hybrid learning process for the knowledge base of a fuzzy rule-based system. In Proceedings of the Information Processing and Management of Uncertainty in Knowledge-Based Systems IPMU 2004, Perugia, Portugal, 2004.

[3] P. Davidsson and M. Boman. Distributed monitoring and control of office buildings by embedded agents. Information Sciences 171 (2005) 293-307, (171):293-307, 2005.

[4] Consejo Superior de Colegios de Arquitectos de España. CTE-HE ahorro de energía, Aplicación a edificios de uso residencial vivienda-DAV. Consejo Superior de Colegios de Arquitectos de España, 2006. ISBN: CTE 84-934051-7-5.

[5] Ministerio de la Presidencia. Real Decreto 1027/2007, de 20 de julio, por el que se aprueba el Reglamento de Instalaciones Térmicas en edificios. http://www.boe.es, BOE num 207, Agosto 2007.

[6] Ministerio de la Vivienda. Real Decreto 314/2006, de 17 de marzo, por el que se aprueba el Código Técnico de la Edificación. 2006. ISBN: 84-340-1641-9. 
[7] Ministerio de Vivienda. Documento Básico de Ahorro de Energia. Limitación de la demanda de Energía. Dirección General de Arquitectura y Polititica de Vivienda, 2005.

[8] J. H. Holland. Escaping Brittleness: The Possibilities of General-Purpose Learning Algorithms Applied to Parallel Rule-Based Systems, volume 2. Morgan Kaufmann, Los Altos, CA, 1986.

[9] V. Julián and V. Botti. Developing real-time multi-agent systems. Integrated Computer-Aided Engineering, 11(2):135-149, 2004.

[10] P. Kinney. Zigbee technology: Wireless control that simply works. Technical report, The ZigBee Alliance, 2007. http://www.zigbee.org/.

[11] P.T. Lewis and D. K. Alexander. Htb2: A flexible model for dynamic building simulation. Building and Environment, (1):7-16, 1990.

[12] The Foundation of Intelligent Physical Agents. The FIPA official site, 2008. http://www.fipa.org/.

[13] J. R. Villar, E.A. de la Cal, and J.Sedano. A fuzzy logic based efficient energy saving approach for domestic heating systems. Integrated Computer-Aided Engineering, 2008. Submitted.

[14] J.R. Villar, E.A. de la Cal, and J. Sedano. Energy saving by means of fuzzy systems. Lecture Notes in Computer Science, 4881:155-161, 2007.

[15] J.R. Villar, E.A. de la Cal, and J. Sedano. Energy savings by means of multi agent systems and fuzzy systems. In Proceedings of the 6th International Workshop on Practical Applications of Agents and Multiagent Systems IWPAAMS 2007, pages 119-128, Salamanca, Spain, 2007.

[16] J.R. Villar, E.A. de la Cal, and J. Sedano. Energy saving by means of fuzzy systems. Lecture Notes in Artificial Intelligence, 5271:583-590, 2008. 